\title{
Identification of inhibitors of ribozyme self-cleavage in mammalian cells via high-throughput screening of chemical libraries
}

\author{
LAISING YEN, ${ }^{1}$ MAXIME MAGNIER, ${ }^{1}$ RALPH WEISSLEDER, ${ }^{2}$ BRENT R. STOCKWELL, ${ }^{3}$ \\ and RICHARD C. MULLIGAN ${ }^{1}$ \\ ${ }^{1}$ Department of Genetics, Harvard Medical School, and Division of Molecular Medicine, Children's Hospital, Boston, \\ Massachusetts 02115, USA \\ ${ }^{2}$ Center for Molecular Imaging Research, Massachusetts General Hospital, Harvard Medical School, Charlestown, \\ Massachusetts 02129, USA \\ ${ }^{3}$ Department of Biological Sciences and Department of Chemistry, Fairchild Center, Columbia University, New York, \\ New York 10027, USA
}

\begin{abstract}
We have recently described an RNA-only gene regulation system for mammalian cells in which inhibition of self-cleavage of an mRNA carrying ribozyme sequences provides the basis for control of gene expression. An important proof of principle for that system was provided by demonstrating the ability of one specific small molecule inhibitor of RNA self-cleavage, toyocamycin, to control gene expression in vitro and vivo. Here, we describe the development of the high-throughput screening (HTS) assay that led to the identification of toyocamycin and other molecules capable of inhibiting RNA self-cleavage in mammalian cells. To identify small molecules that can serve as inhibitors of ribozyme self-cleavage, we established a cell-based assay in which expression of a luciferase (luc) reporter is controlled by ribozyme sequences, and screened 58,076 compounds for their ability to induce luciferase expression. Fifteen compounds able to inhibit ribozyme self-cleavage in cells were identified through this screen. The most potent of the inhibitors identified were toyocamycin and 5-fluorouridine (FUR), nucleoside analogs carrying modifications of the 7-position and 5-position of the purine or pyrimidine bases. Individually, these two compounds were able to induce gene expression of the ribozyme-controlled reporter $\sim 365$-fold and 110 -fold, respectively. Studies of the mechanism of action of the ribozyme inhibitors indicate that the compounds must be incorporated into RNA in order to inhibit RNA selfcleavage.
\end{abstract}

Keywords: gene regulation; ribozyme self-cleavage; ribozyme inhibitors; toyocamycin; 5-fluorouridine

\section{INTRODUCTION}

The ability to "exogenously" control the expression of genes in mammalian cells has been a powerful tool of biomedical research (Gossen and Bujard 2002). Gene regulation technology has already played a critical role in efforts to understand the role of specific gene products in fundamental biological processes, in development, and in disease states. In the future, the technology offers the opportunity to have a major impact in new areas of research and medicine. To date, most commonly used gene regulation systems rely on the control of transcription

Reprint requests to: Richard C. Mulligan, Harvard Gene Therapy Initiative, Harvard Institutes of Medicine, Suite 407, 4 Blackfan Circle, Boston, MA 02115, USA; e-mail: mulligan@receptor.med.harvard.edu; fax: (617) 432-6146.

Article published online ahead of print. Article and publication date are at http://www.rnajournal.org/cgi/doi/10.1261/rna.2300406.
(Gossen and Bujard 1992; Wang et al. 1994; Rivera et al. 1996; Suhr et al. 1998). More recently, the discovery of RNA interference and siRNAs has led to the development of novel strategies for controlling gene expression that rely on the targeting of RNA (Fire et al. 1998; McManus and Sharp 2002). In the hopes of extending the application and utility of gene regulation technology, we have been interested in the development of new methods for controlling gene expression that rely on the modulation of RNA selfcleavage rather than transcription. The basis for such a system is the incorporation of sequences encoding selfcleaving RNA motifs (i.e., ribozyme sequences) into a mammalian transcription unit, such that upon generation of the resulting mRNA, spontaneous ribozyme self-cleavage should lead to destruction of the mRNA and therefore no gene expression. Administration of agents capable of inhibiting RNA self-cleavage should result in preservation 
of the intact mRNA and therefore reinstate (i.e., "induce") gene expression (Fig. 1A).

The two essential requirements of such an RNA-only gene regulation system are the availability of ribozyme sequences capable of efficient self-cleavage in mammalian cells when embedded in an mRNA transcript, and the availability of small molecules capable of inhibiting such self-cleavage in mammalian cells. With regard to the first requirement, we have recently tested a large number of natural and synthetic ribozyme sequences for their ability to function in mammalian cells when embedded in an mRNA transcript. We were able to identify and further develop a modified version of a hammerhead ribozyme, derived from Schistosoma mansoni (Sm1), that is capable of extremely efficient self-cleavage in mammalian cells (Yen et al. 2004). This ribozyme, termed N79, was found to function in a variety of commonly used mammalian cell lines and in primary cells in vivo. An important proof of principle for the use of inhibitors of RNA self-cleavage to control gene expression was provided by the demonstration of the ability of one specific small molecule inhibitor of RNA self-cleavage, toyocamycin, to control gene expression in vitro and vivo. Here, we describe the development of the high-throughput screening (HTS) assay that led to the identification of toyocamycin and other inhibitors of RNA self-cleavage. Rather than rely on in vitro screens of compounds, we chose to develop a mammalian cell-based screen in order to directly identify molecules capable of functioning within cells. Since a variety of aminoglycoside (Stage et al. 1995; Murray and Arnold 1996; Hermann and Westhof 1998; Tor et al. 1998; Jenne et al. 2001) and nonaminoglycoside antibiotics (Jenne et al. 2001) had been previously shown to be able to inhibit the self-cleavage of hammerhead ribozymes in the in vitro setting, we first screened such compounds in the cell-based assay. We then extended the studies to include the high-throughput screening of 58,076 compounds. We report here the results of those screening efforts and the characteristics (and in some cases, the mechanism of action) of the inhibitors that were identified.

\section{RESULTS}

\section{Generation of reporter cell lines for cell-based screening}

For screening purposes, we chose to generate a stable human (HEK-derived) cell line that carries an integrated mammalian expression vector in which a luc reporter gene's expression is placed under the control of the CMV promoter and with two copies of the N79 ribozyme (Fig. 1B). Stable cell lines were generated by cotransfection of HEK 293 cells with the reporter construct, an expression
A

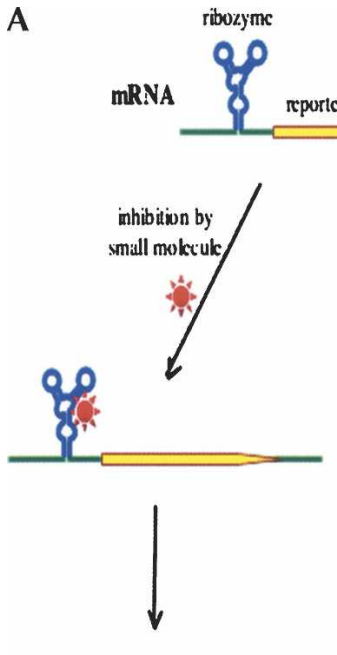

induced genc expression

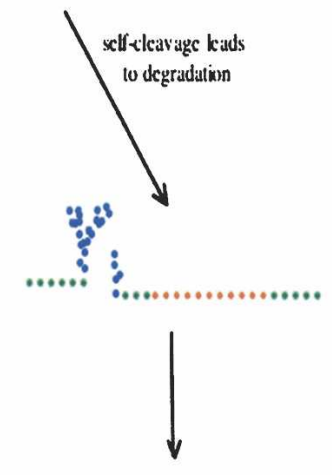

n) genc cxpression
B

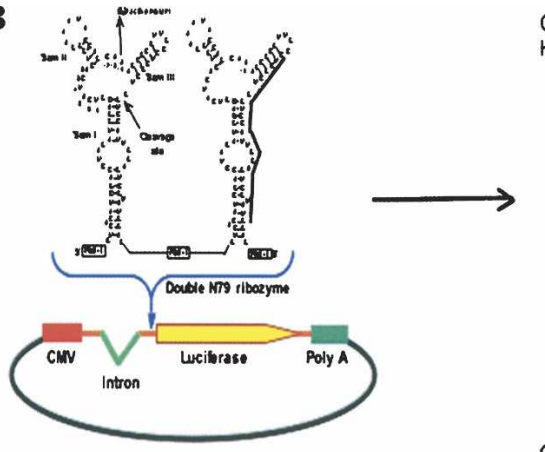

Generation of stably tanstected HEK293 cells clones

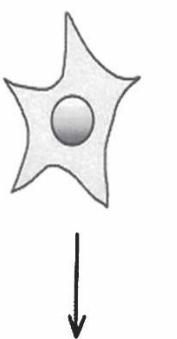

Choose clones on bas is of inducibility ol luciferase after addition of anisense morphoino oligo

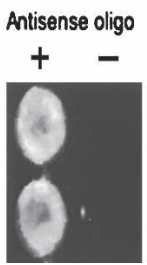

FIGURE 1. Strategy for identifying new ribozyme inhibitors via high-throughput screening. $(A)$ Controlling gene expression via the modulation of RNA self-cleavage. When a cis-acting hammerhead ribozyme is embedded in the mRNA, self-cleavage leads to the destruction of the mRNA and results in the absence of gene expression. However, the administration of specific inhibitors of the ribozyme leads to the generation of intact mRNAs and results in protein expression. (B) Schematic representation of the N79 ribozymes and the reporter gene expression vector used for the cell-based screening assay. Stable cell clones that had low background luc expression and could be induced by morpholino anti-sense oligonucleotides as shown in the bioluminescent images were selected for the use in the screen. The black line identifies the sequence targeted by the anti-sense oligonucleotide. Nucleotide numbering follows standard nomenclature (Hertel et al. 1992). 
vector encoding puromycin resistance, and subsequent selection in puromycin-containing medium. Approximately 10 clonal cell lines were generated in this way. To identify specific cell lines capable of responding to putative inhibitory molecules, all cell lines were tested for both their basal level of luc expression and for the extent of induction of luc expression achieved after transfection of the cells with an anti-sense morpholino oligonucleotide known to block the cleavage of N79 (Yen et al. 2004). Several cell lines tested in this way expressed very low basal levels of luc, yet were readily induced to express luc after administration of oligonucleotide. One of these cell lines, termed HEK79, showed a 20-fold induction of luc expression after oligonucleotide administration and was chosen for high-throughput screening (Fig. 1B).

\section{High-throughput screening of compound libraries}

As indicated above, several different antibiotic compounds have been previously shown to inhibit ribozyme activity in vitro. As a first step toward the identification of compounds capable of inhibiting ribozyme cleavage in mammalian cells, we tested a variety of such compounds (Table 1), using both the HEK79 cell line and cells transiently transfected with different N79 variants. None of the compounds tested revealed an appreciable inhibitory effect on ribozyme self-cleavage, when administered at concentrations of either 10 or $100 \mu \mathrm{M}$ (data not shown).

Based on those results, we extended the screening effort to include 58,076 compounds. The full set of compounds included an annotated bioactive compound library (2036 compounds) (Root et al. 2003); a diversity library obtained from NINDS (1040 compounds) (Lunn et al. 2004); a collection of FDA-approved drugs (1000 compounds) (Rogers et al. 2002); a combinatorial library from Comgenex (20,000 compounds); a library containing both natural products and synthetic compounds (TIC; 24,000 compounds) (Kelley et al. 2004); and a combinatorial library called the "World Diversity Set," obtained from Specs, containing compounds chosen for geographic as well as

TABLE 1. Survey of ability of different antibiotics to inhibit hammerhead ribozyme function

Chlortetracycline

Demeclocycline

Diminazene aceturate

Distamycin A

Doxorubicin

5-epi-Sisomicin

Gentamicin sulfate

G418

Gramicidin D

Hoechst 33258

Hoechst 33342 chemical diversity (10,000 compounds). For this highthroughput screening effort, the HEK79 cells were seeded in a 386-well format and exposed to the different compounds at a final concentration of $4 \mu \mathrm{g} / \mathrm{mL}$ for $48 \mathrm{~h}$, and luc activity was then measured using a plate reader. Molecules were considered positive if they induced luc expression in cells carrying functional N79 ribozymes but not in cells carrying inactive N79 ribozymes. Through this primary screening effort and subsequent analysis of similar analogs of positive molecules, 15 ribozyme inhibitors were identified. The maximal fold increase in luc expression achieved by each compound (Foldmax) and the concentration that yields $50 \%$ of maximal induction (EC50) are summarized in Table 2. All of the identified molecules were shown to possess comparable activity in a separately generated N79-carrying cell line to that observed in the HEK79 cell line used for the original screening (data not shown). Interestingly, none of the inhibitors that were identified were aminoglycosides, despite the presence of many such molecules in the libraries.

\section{Characterization of positive compounds}

Based on structural similarities, the identified molecules can be divided into three groups. The first group consists of adenosine analogs, including tubercidin (7-deaza-adenosine), tubercidin-monophosphate and -triphosphate, tubercidincyclic monophosphate, sangivamycin, 8-azaadenosine, tricyclic nucleoside, and toyocamycin. All members of this group contain at least a modified 7-position on the purine (Fig. 2A). Analogs that have modifications other than at the 7-position, such as 2-amino, 3-deaza, 6-chloro, 9-deaza, lacked an inhibitory effect. In addition, the identified compounds all contain a $2^{\prime} \mathrm{OH}$ group on the sugar base, indicating that a ribose is necessary for their inhibitory effects (Fig. 2A). Similar analogs that lack $2^{\prime} \mathrm{OH}$ or are missing the ribose entirely produced no appreciable inhibition (2'-deoxy-tubercidin, 2'-deoxy-toyocamycin) (Fig. 2B). Of all the compounds, toyocamycin (Aszalos et al. 1966) was found to be the most potent ribozyme inhibitor, resulting in up to 365 -fold luc induction when applied to the stable cell line. The effectiveness of the remaining compounds in this group was $<60$-fold (Table 2). Based on its apparent higher effectiveness, a dose-response curve of toyocamycin on the stable cell line was generated. As shown in Figure $2 \mathrm{C}$, the induction peaked rapidly around $1 \mu \mathrm{M}$ and then dropped off sharply. This drop coincided with apparent cell toxicity observed by microscopy. In addition, a decrease of luc expression from cells carrying inactive ribozymes, presumably due to the toxicity effects of the compound, was observed when the concentration of toyocamycin reached $0.5 \mu \mathrm{M}$. The dose-limiting toxicity of toyocamycin thus excludes its use at higher concentration or for longterm applications. 
TABLE 2. Compounds found to inhibit ribozyme activity in cells

\begin{tabular}{lcrl}
\hline Compound name & $\mathrm{EC}_{50}(\mu \mathrm{M})^{\mathrm{a}}$ & Fold $_{\max } \mathrm{b}$ & Class \\
\hline Toyocamycin & 0.4 & 378.2 & Adenosine analog \\
8-Azaadenosine & 3.6 & 45.4 & Adenosine analog \\
Sangivamycin & 1.1 & 38.2 & Adenosine analog \\
Tubercidin & 2.5 & 57.8 & Adenosine analog \\
Tubercidin-cyclic monophosphate & 34.4 & 35.1 & Adenosine analog \\
Tubercidin-monophosphate & 2.2 & 39.3 & Adenosine analog \\
Tubercidin-triphosphate & 1.9 & 34.7 & Adenosine analog \\
Nebularine & 10.2 & 8.4 & Adenosine analog \\
Tricyclic nucleoside & 39.5 & 12.0 & Adenosine analog \\
5-Fluoro-uridine & 5.9 & 120.9 & Uridine analog \\
5-Bromo-uridine & 266.6 & 15.5 & Uridine analog \\
5-Fluoro-uracil & 499.5 & 65.3 & Uridine analog \\
Syto-83 & 7.4 & 25.6 & RNA-binding dye \\
Homidium bromide & 2.3 & 8.4 & RNA-binding dye \\
Acridine orange & 7.0 & 3.6 & RNA-binding dye \\
\hline
\end{tabular}

${ }^{a}$ The effective concentration at $50 \%$ of maximal induction.

bumbers are maximal "fold increase" in luciferase expression after $24 \mathrm{~h}$ of compound treatment.
The second group of ribozyme inhibitors identified in the screen consists of uridine analogs, including 5-bromouridine, 5-fluorouridine (FUR), and 5-fluorouracil (5FU) (Fig. 3A). All contain a 5-position modification on the pyrimidine. Analogs that contain an iodo, a methyl, a hydroxyl, an ethyl, or an aza group on the 5-position lack an inhibitory effect. In the case of this group of inhibitors, FUR was the most potent. However, replacing the $2^{\prime} \mathrm{OH}$ group of FUR by either $2^{\prime}$ deoxy or 2 ' O-methyl, or by inverting the stereocenter of the $2^{\prime}$-position (Fig. 3B), resulted in loss of inhibition, indicating that, as with adenosine analogs, a ribose is necessary for the inhibitory effects of uridine analogs. In contrast to toyocamycin, the dose-response curve of FUR reached a plateau of 110 -fold at $50 \mu \mathrm{M}$, and the plateau was maintained for a wide range of concentrations (Fig. 3C), suggesting that an efficient induction can be reached with a lower dosage before reaching severe toxicity. The related molecule 5FU (Longley et al. 2003), however, has a doseresponse curve resembling that of toyocamycin, reaching a peak of 65 -fold followed by a quick drop-off (Fig. 3D). The effectiveness of 5FU was also significantly less, requiring a concentration of $500 \mu \mathrm{M}$ to reach $50 \%$ of the maximal induction, as compared to the $6 \mu \mathrm{M}$ required for FUR (see EC50 in Table 2). For both FUR and 5FU, a decrease of luc expression from cells carrying inactive ribozymes was observed when concentrations reached 800 $\mu \mathrm{M}$.

The third group of ribozyme inhibitors belongs to the family of RNA-binding dyes, and includes Syto-83, ethidium bromide (homidium bromide), and acridine orange (Fig. 4A). These dyes were also far less potent inhibitors than the adenosine or uridine analogs (Table 2).

As mentioned earlier, the N79 ribozyme used in our cellbased assay was developed from Sm1 of S. mansoni
(Ferbeyre et al. 1998), a human parasite that infects people in many areas of the world (Ross et al. 2002). It is intriguing that one of the drugs suggested in the past as potential treatments for Schistosome infection was tubercidin (Jaffe et al. 1971; el Kouni 1991), the same molecule identified in our screen as an Sml ribozyme inhibitor. This prompted us to test other molecules with known anti-Schistosomal effects for their inhibitory potential. These included nebularine, dipyridamole, benzylacyclouridine, metrifonate, oxamniquine, praziquantel, and artemether. However, only nebularine (also an adenosine analog) (el Kouni and Cha 1987) showed an inhibitory effect on ribozyme selfcleavage, albeit minimal (Table 2). Interestingly, unlike most of the adenosine analogs found in our screen, nebularine contains a modification on the 6- but not the 7-position of the purine base (Fig. 2A).

Lastly, it should be noted that while there were numerous guanosine and cytidine analogs in the 58,076 compounds screened, none of them surfaced as ribozyme inhibitors. In particular, 7-deazaguanine and 7-deazaguanosine triphosphate (the purine counterparts of tubercidin) and 5Fcytosine (the pyrimidine counterpart of 5FU) (Fig. 4B) all showed no inhibitory effects when tested at similar concentrations that produced inhibition by adenosine analogs or uridine analogs. The fact that only adenosine and uridine analogs but no guanosine and cytidine analogs were found positive may provide an insight into the N79 structurefunction relationship (see below).

\section{Mechanisms responsible for ribozyme inhibition: RNA incorporation vs. RNA binding}

To examine the mechanisms responsible for ribozyme inhibition, we incubated the N79 ribozyme with either tubercidin or toyocamycin in an in vitro catalytic reaction. Surprisingly, neither tubercidin (Fig. 5A, lanes 3-8) nor toyocamycin (Fig. 5B, lanes 3-8) was able to inhibit ribozyme self-cleavage in vitro, as indicated by the unaltered cleavage percentage over a broad range of compound concentrations. The lack of direct inhibition by these molecules indicated that their mechanism of inhibition was not likely due to direct ribozyme-compound interactions. In light of our findings that inhibitory activity appeared to always be associated with a $2^{\prime} \mathrm{OH}$ group on the sugar base, we next asked whether covalent incorporation of these analogs into the ribozyme-containing RNA might be required for the inhibition of RNA self-cleavage. To test if covalent incorporation of identified inhibitory molecules can, indeed, lead 


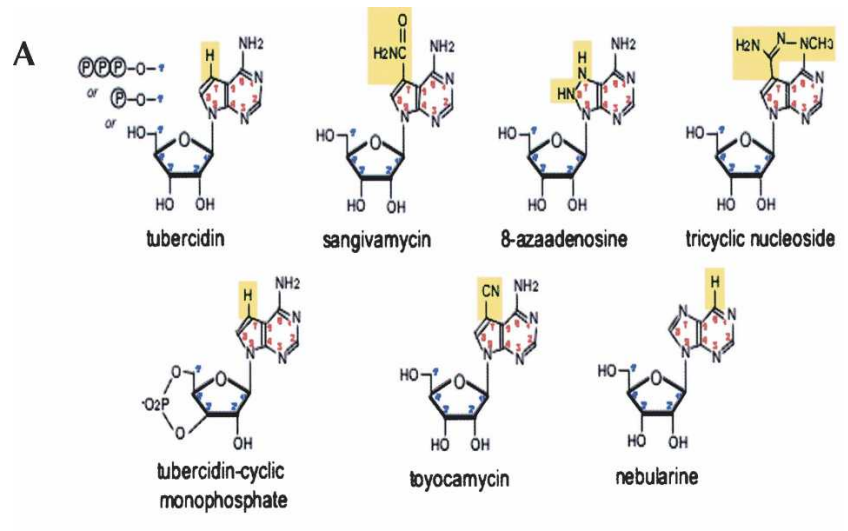

B

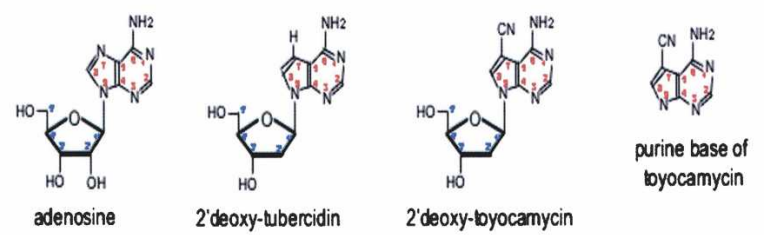

C

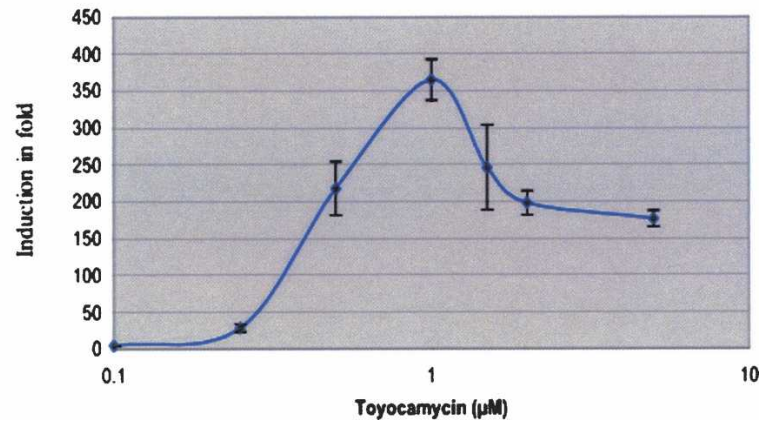

FIGURE 2. Chemical structures of identified adenosine analog ribozyme inhibitors and related compounds. (A) Analogs capable of inhibiting ribozyme self-cleavage in cells contain at least a modified 7-position on the purine, with the exception of nebularine, which contains a 6-position modification. These compounds all have a $2^{\prime} \mathrm{OH}$ group on the sugar base. The differences between these compounds and the inactive adenosine are indicated by the yellow shade. (B) Examples of analogs that lacked an inhibitory effect. (C) Doseresponse curve of toyocamycin in log scale. Error bars indicate 1 S.D. (ㅁandard deviation).

to cleavage inhibition, tubercidin-triphosphate was used in place of ATP in an in vitro transcription reaction to generate ribozyme RNA carrying substitutions in all adenosine positions. As shown in Figure 5C, this substitution resulted in the complete loss of ribozyme self-cleavage (Fig. 5C, lanes 2-6), while the unsubstituted ribozyme cleaved efficiently under the same conditions (Fig. 5C, lane 7).

Similarly, FUR (Fig. 5D) and 5FU (Fig. 5E) exhibited no inhibitory effect when incubated directly with N79 ribozyme at concentrations that would normally produce pronounced induction in the cell-based assays. Our attempts to examine the effect of incorporating FUR into the N79 ribozyme were inconclusive due to the difficulty of generating full-length FUR-substituted N79 ribozyme by T7 polymerase. However, past studies using chemical synthesis to generate FUR-containing hammerhead ribozymes demonstrated that when incorporated at certain key positions of the ribozyme, FUR, indeed, impaired self-cleavage (Hammann et al. 2001).

A

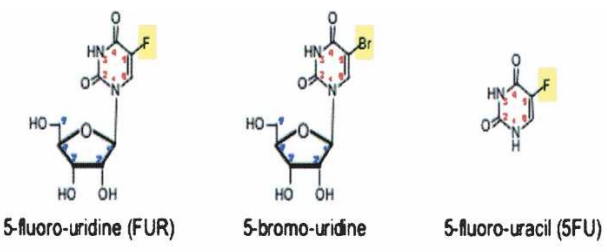

B
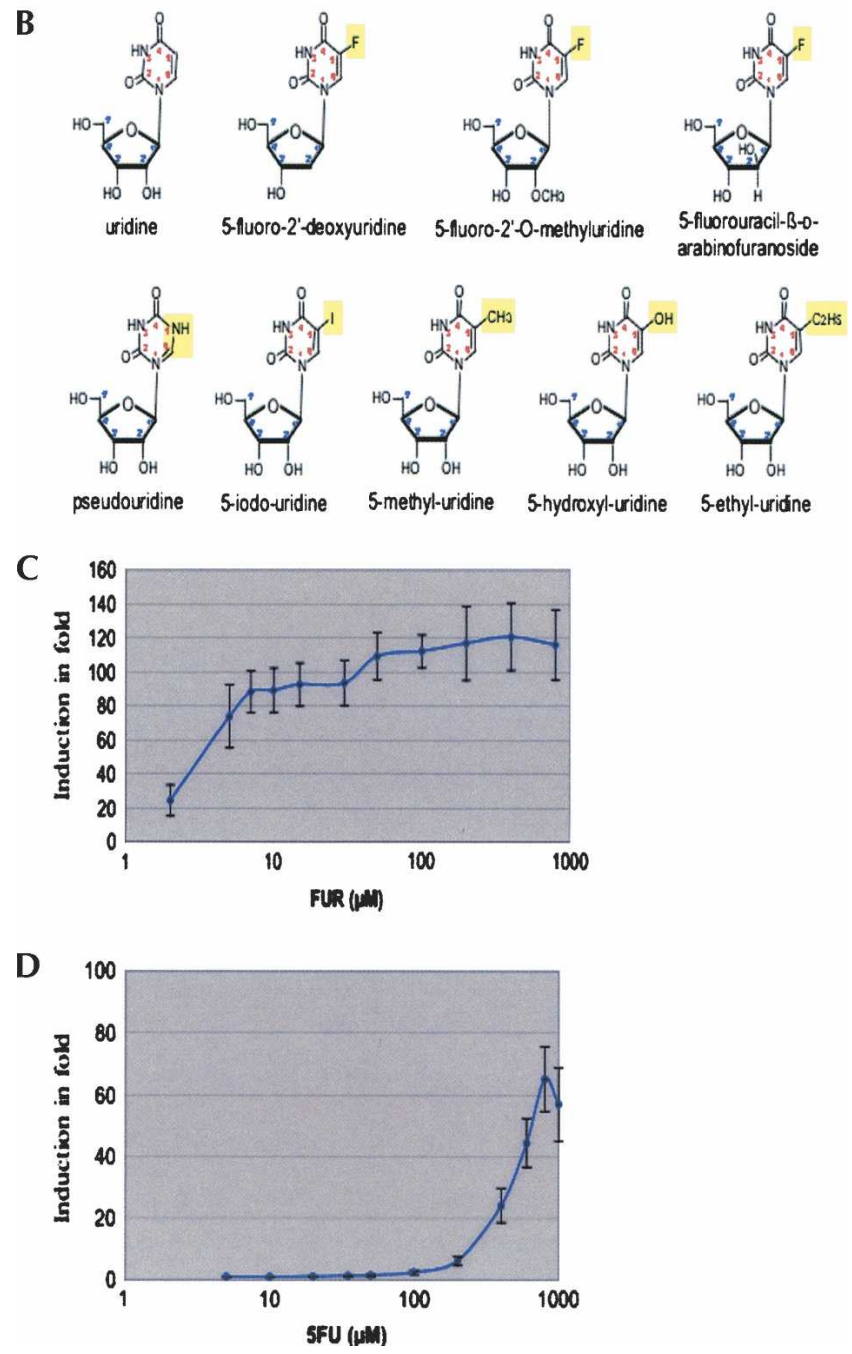

FIGURE 3. Chemical structures of identified uridine analog ribozyme inhibitors and related compounds. (A) Analogs capable of inhibiting ribozyme self-cleavage in cells contain a modified 5position on the pyrimidine. These compounds all have a $2^{\prime} \mathrm{OH}$ group on the sugar base. The differences between these compounds and the inactive uridine are indicated by the yellow shade. (B) Examples of analogs that lacked the inhibitory effect. $(C)$ Dose-response curve of FUR in log scale. $(D)$ Dose-response curve of 5FU in log scale. Error bars indicate 1 S.D. 
A
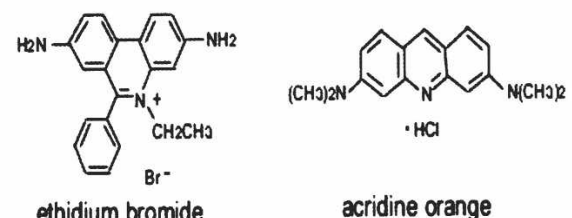

acridine orange

B

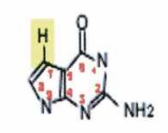

7-deazaguanine

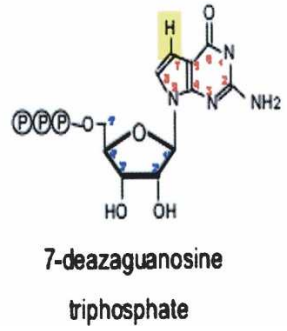

FIGURE 4. Chemical structures of identified RNA-binding dye ribozyme inhibitors and several guanine and cytidine analogs related to tubercidin and 5FU that lack inhibitory activity. (A) RNA-binding dyes. The structure of Syto- 83 is currently unavailable. (B) Structure of guanosine and cytidine analogs with similar functional groups to that of tubercidin and 5FU but that lacked the inhibitory activity.

Consistent with the mechanism of covalent incorporation of the compounds into the ribozyme-encoding cellular mRNA, it has been reported that tubercidin (Acs et al. 1964), toyocamycin (Suhadolnik et al. 1967), 5-FUridine (Glazer and Lloyd 1982), and 5-FU (Glazer and Lloyd 1982) are able to enter the ribonucleotide pool for RNA synthesis and can be found in the cellular RNAs of mammalian cells. This mechanism of inhibition of ribozyme function, dependent on inhibitor incorporation into RNA, may shed light on the basis for our identification of only adenosine analogs with a modified 7-position and uridine analogs with a modified 5-position as effective inhibitors and our inability to identify any analogous guanosine and cytidine analogs. While these findings might simply relate to the variable cell permeability of different molecules, or their ability to be recognized by RNA polymerases for incorporation, they may suggest that structural interactions involving the 7- and 5-positions of $\mathrm{A}$ and $\mathrm{U}$ bases, respectively, are particularly important for N79 ribozyme function in vivo. Although the 7-position of A does not form hydrogen bonds in the standard Watson-Crick base pairs, the X-ray structures of hammerhead ribozymes, indeed, showed its involvement in forming the nonstandard A-G base pairs in the conserved core (A9-G12, A13-G8; see Fig. 2 for the nucleotide numbering) (Fu and McLaughlin 1992; Pley et al. 1994; Scott et al. 1995). The role of the 5-position of $\mathrm{U}$ is less clear since it is not involved in hydrogen bonds of either the standard Watson-Crick or the G-U wobble base pairs. Nevertheless, it was shown that incorporating FUR at the U4 and U7 of the hammerhead core led to the impairment of self-cleavage (Hammann et al. 2001). Thus, modifying those $\mathrm{A}$ and $\mathrm{U}$ bases in the conserved core could lead to the graded loss of cleavage activity in vivo. Furthermore, the N79 ribozyme contains an unusually high percentage of A and U bases in its loops I and II. Substituting these bases could disrupt the critical interaction of the two loops required for the ribozyme to function in cells (De la Pena et al. 2003; Khvorova et al. 2003; Penedo et al. 2004; Yen et al. 2004).

\section{Synergistic inhibition of ribozyme function by combined administration of toyocamycin and FUR}

As toyocamycin (an adenosine analog) and FUR (a uridine analog) would occupy different positions if they were incorporated into the N79 ribozyme, we next asked whether combined administration of the two compounds might lead to improved inhibition of ribozyme function relative to addition of a single compound and perhaps define a less toxic regimen for achieving reasonable levels of induction of gene expression. To address this question, varying amounts of each compound were combined and added to HEK79 cells, and subsequently, the fold induction of luc expression was determined. As shown in Figure 6, administration of specific concentrations of the two compounds did, indeed, result in a more efficient inhibition of the ribozyme than was possible with either compound alone (e.g., 670-fold induction by a combination of the two compounds vs. 120 -fold induction by FUR alone or 378-fold induction by toyocamycin alone). In addition, a variety of mixtures of the two compounds were able to induce gene expression between 200- and 600-fold (Fig. 6), in contrast to the narrow range of concentrations of toyocamycin for which induction can be achieved in the absence of significant toxicity (Fig. 2C). To document the practical use of this two-compound cocktail for the regulation of gene expression in vivo, we established an in vivo model in which an AAV vector encoding a luc reporter controlled by two copies of the N79 ribozyme (similar transcription cassette to that diagrammed in Fig. 1) was injected subcutaneously to the left ear of nude mice. To provide an internal control, all mice were also injected in the right ear with virus carrying the inactive N79. After $3 \mathrm{wk}$, these mice were imaged for basal luc gene expression (day 0), and immediately after imaging, a single pulse of cocktail was of mice, taken before and 2-3 d after cocktail injection, are shown in Figure 7. The images demonstrate that, as expected, the right ear carrying the inactive N79 showed little change in gene expression before and after cocktail treatment. In contrast, in the left ear carrying the functional N79, a pronounced induction was readily detectable after the treatment. Quantification of the photon output indicated that the induced gene expression reached 37-, 17-, and 13fold in the three mice treated. The induction afforded by this single pulse injection method was somewhat variable from mouse to mouse, most likely due to the variability of cocktail volume delivered. 

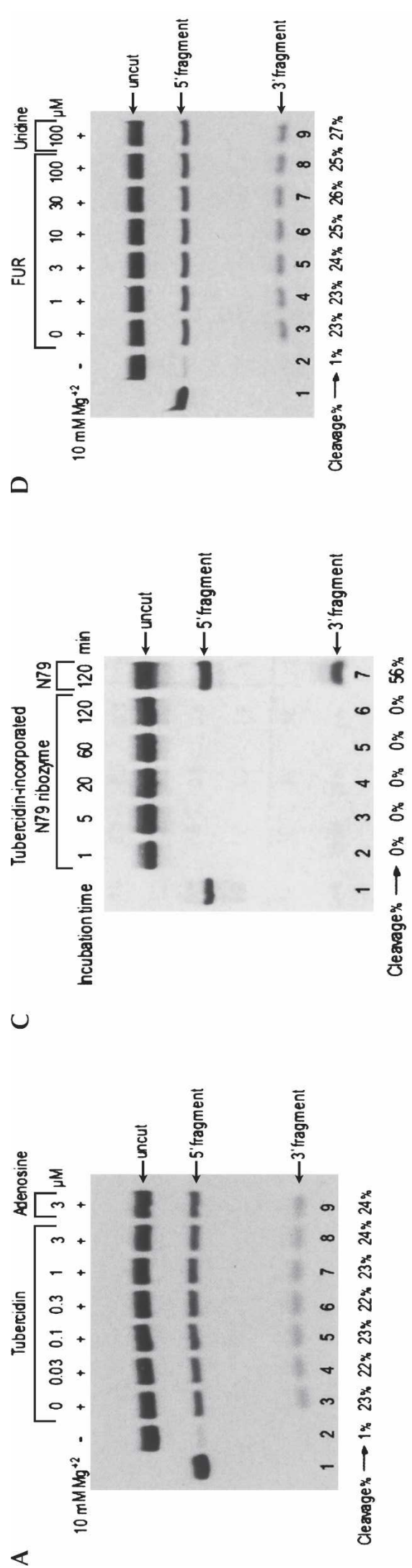

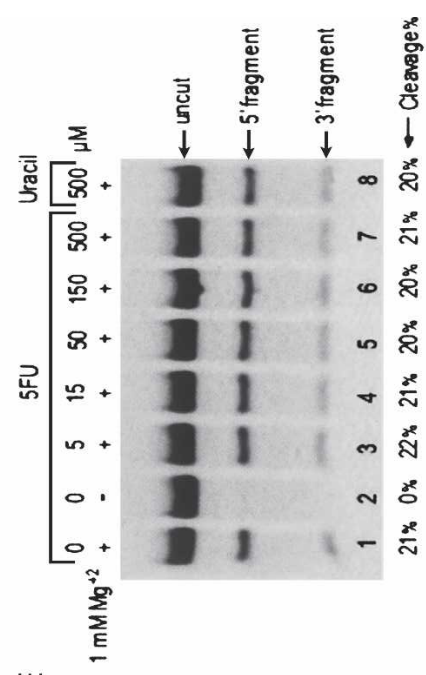

ш

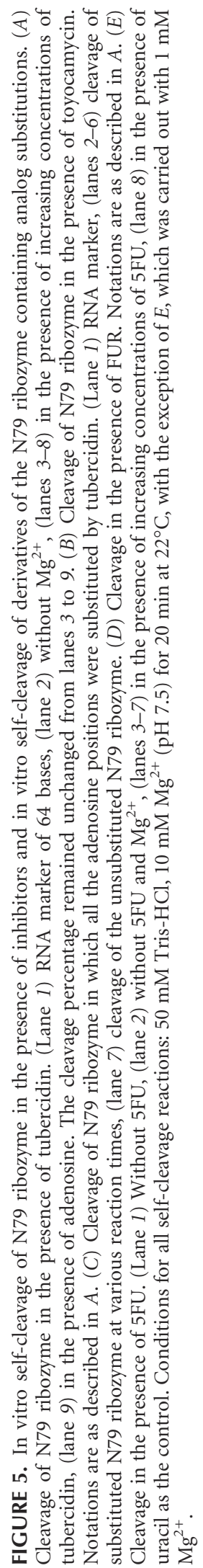




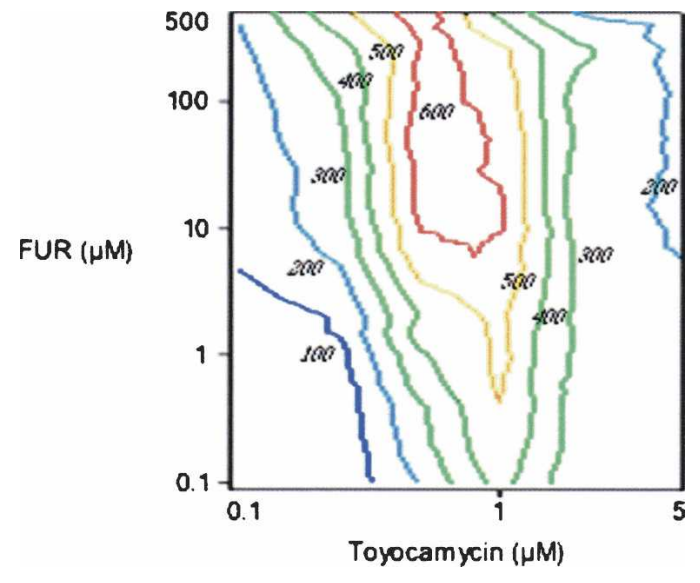

FIGURE 6. Combined administration of toyocamycin and FUR leads to improved levels of induction of gene expression. The numbers next to each contour line represent "fold increase" in gene expression induced by $24 \mathrm{~h}$ of cocktail treatment. The concentrations are in log scale.

\section{DISCUSSION}

The work described here represents the first cell-based screen aimed at identifying small molecules capable of modulating ribozyme self-cleavage in vivo. By screening 58,076 compounds, we were able to identify two small molecules, toyocamycin and FUR, that can potently inhibit ribozyme function in mammalian cells. It is of significant interest that the two most potent inhibitors of ribozyme function in cells that we identified appear to inhibit ribozyme
Day 0

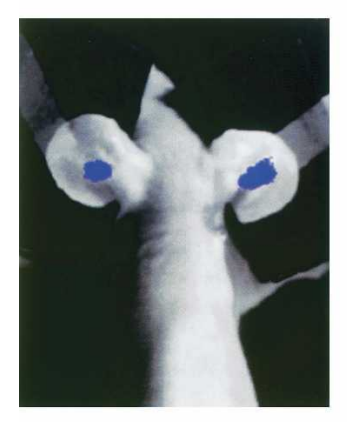

Day 2

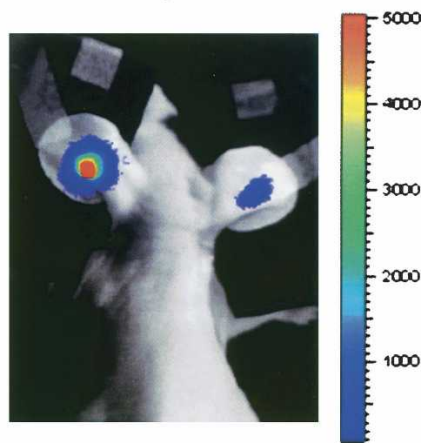

FIGURE 7. Control of gene expression in vivo after coadministration of toyocamycin and FUR. About $5 \mu \mathrm{L}$ of recombinant AAV carrying the active N79/luc transgene (titer of $10^{12}$ genome copies $/ \mathrm{mL}$ ), or recombinant AAV carrying the inactive N79/luc transgene (titer of $5 \times 10^{9}$ genome copies $/ \mathrm{mL}$ ) were injected into the left or the right ear, respectively. Three weeks later, a single pulse of roughly $5 \mu \mathrm{L}$ of cocktail containing $20 \mu \mathrm{M}$ of toyocamycin and $12 \mathrm{mM}$ of FUR was injected in each ear (day 0). Animals were imaged as previously described (Yen et al. 2004) to determine the levels of luc expression prior to the addition of compounds, and $2 \mathrm{~d}$ after compound treatment. The figure shows a representative animal of the threeanimal cohort. The induction in fold described in text was calculated based on the photon output in the left ear before and after cocktail treatment, and was normalized to the photon output from the right ear. self-cleavage via their covalent incorporation into the mRNA carrying the ribozyme sequences, as evidenced by the inability of these molecules to inhibit ribozyme selfcleavage in vitro and by the loss of self-cleavage activity when these molecules are incorporated. Based on the welldescribed interactions between specific aminoglycosides and other small molecules and RNA (Stage et al. 1995; Murray and Arnold 1996; Hermann and Westhof 1998; Tor et al. 1998; Jenne et al. 2001), we had expected that our extensive screen of compounds would have led to the identification of compounds that inhibit ribozyme selfcleavage by virtue of their direct binding to ribozymecontaining RNA. Our inability to identify such compounds may suggest that the complex structure of mRNA molecules and their associated proteins present in mammalian cells greatly restricts the possibilities for the types of interactions between small molecules and RNAs that can lead to the inhibition of RNA self-cleavage. The ability of morpholino oligonucleotides and several RNA-binding dyes to inhibit ribozyme activity in cells, however, suggests that appropriate interactions between specific small molecules and ribozymes that lead to inhibition of self-cleavage in vivo are indeed possible. We are hopeful that additional screening efforts will lead to the identification of new inhibitors of ribozyme function with improved toxicity profiles and more desirable pharmacokinetic properties.

We had already made use of one of the molecules identified in the current study, toyocamycin, to provide a critical "proof of principle" for the ability to regulate gene expression in mammalian cells both in vitro and in vivo via the modulation of RNA self-cleavage (Yen et al. 2004). As expected by the proposed mode of action of this inhibitor, we showed in that study that in the absence of toyocamycin, the ribozyme-controlled mRNA was not detectable in the nucleus or cytoplasm in cells carrying active ribozymes, consistent with the mechanism that ribozyme self-cleavage leads to rapid degradation of mRNAs. However, when these cells were treated with toyocamycin, the amount of luc mRNA increased to a level comparable to that of cells carrying inactive ribozyme, which is consistent with the mechanism that inhibition of ribozyme self-cleavage leads to the generation of intact mRNA in cells (Yen et al. 2004). In the present studies, we show that the combined administration of toyocamycin and FUR to cells harboring a ribozyme-regulatable expression construct leads to an improved induction of gene expression, relative to the use of toyocamycin alone. This combined regimen requires lower concentrations of the two compounds than must be used to achieve reasonable levels of inductions using either compound alone, and consequently results in a less toxic effect on the cells. While it is hopeful that the use of such a two-compound cocktail may enable more experimental applications of the ribozyme-based gene regulation system than are possible with toyocamycin alone, the identification 
of significantly less toxic inhibitors of ribozyme function remains an important goal of future research.

In addition to the use of the ribozyme-based gene regulation system for the control of expression of gene products, the finding that one of the most commonly used chemotherapeutic agents, $5 \mathrm{FU}$, can serve as a ribozyme inhibitor in vivo raises the interesting possibility that one novel application of RNA-only gene regulation systems might relate to the real-time "sensing" of the presence of specific compounds in vivo. In the specific case of $5 \mathrm{FU}$, the ability of the molecule to induce expression of a reporter gene could provide an important means of monitoring $5 \mathrm{FU}$ uptake and retention by tumors and normal tissues, and/or facilitate efforts to develop more optimal routes of $5 \mathrm{FU}$ administration.

\section{MATERIALS AND METHODS}

\section{High-throughput screening of compound libraries}

ACL, NINDS, TIC, and Comgenex libraries were collected by Dr. Brent Stockwell, prepared as $4 \mathrm{mg} / \mathrm{mL}$ solutions in DMSO, and screened at a final concentration of $4 \mu \mathrm{g} / \mathrm{mL}$. FDA2000 was collected by Dr. Steve Gullans (BWH), and each compound was prepared at $10 \mathrm{mM}$ in DMSO and screened at $10 \mu \mathrm{M}$. The Specs library was prepared as $10 \mathrm{mg} / \mathrm{mL}$ and screened at $10 \mu \mathrm{g} / \mathrm{mL}$.

HEK79 cells were seeded in white, opaque-bottom 386-well plates at a density of 8000 cells per well in medium with $10 \%$ serum and pen/strep using the Zymark dispenser. On the same day, cells were treated with compounds at a final concentration of $4 \mu \mathrm{g} / \mathrm{mL}$ and $0.1 \%$ DMSO, followed by an incubation period of $48 \mathrm{~h}$ at $37^{\circ} \mathrm{C}$ in a humidified incubator containing $5 \% \mathrm{CO}_{2}$. Cells were then lysed, and luciferin was added to the cells at a final concentration of $4 \mu \mathrm{g} / \mathrm{mL}$; the amount of light output from each well was recorded on a Packer Fusion platereader. Induction of luc expression by a particular compound was calculated by subtracting the signal with instrument background and background from untreated cells. All screens were performed in triplicate repeats.

\section{Cell culture assays for testing the effective concentration of compounds}

Plasmid DNAs carrying a LacZ reporter under the control of two copies of the N79 ribozyme were transfected into subconfluent HEK293T cells using Fugene6 (Roche). The next day, cells were treated with compounds at various concentrations for $24 \mathrm{~h}$. The level of $\beta$-galactosidase expression was then quantified by ONPG assay $24 \mathrm{~h}$ later. All experiments were performed at least in triplicate. Alternatively, HEK79 cells (stable cell lines containing a luc reporter under the control of two copies of N79 ribozymes; see Results) were seeded in white, opaque-bottom 96-well plates with a density of 40,000 cells per well, and treated with compounds the next day at various concentrations for $24 \mathrm{~h}$. Luciferin was then added to the cells at a final concentration of $20 \mu \mathrm{g} / \mathrm{mL}$, and the amount of light output from each well was recorded on a plate reader. All experiments were performed at least in triplicate. 5FU (from Sigma) was prepared as $100 \mathrm{mM}$ stock in 100\% DMSO;
FUR (Sigma), as $10 \mathrm{mM}$ stock in PBS; toyocamycin (Berry \& Associates) and tubercidin (Sigma), as $10 \mathrm{mM}$ stock in $10 \%$ DMSO.

\section{In vitro transcription and catalytic reaction}

DNA templates containing a T7 promoter and the ribozyme sequences were generated by PCR. ${ }^{33} \mathrm{P}$-labeled RNA was then transcribed by T7 RNA polymerase in the presence of $50 \mu \mathrm{M}$ blocking anti-sense oligonucleotides using Ambion's MEGAshortscript kit. The anti-sense oligonucleotide sequence used was 5'-GATAGCAGTGGAATCCAGGACGCAC-3'. In the absence of the anti-sense oligonucleotide, $\sim 95 \%$ of the transcripts were cleaved during the 3 - $\mathrm{h}$ in vitro transcription reactions. In the presence of the anti-sense oligonucleotide, the percentage of cleavage products dropped to $35 \%$. To make ribozymes with incorporated tubercidin, transcription was carried out by substituting ATP with tubercidin triphosphate. Full-length RNAs were then purified by $10 \%$ denaturing polyacrylamide gel. To determine the cleavage efficiency, ribozyme was incubated in $50 \mathrm{mM}$ Tris- $\mathrm{HCl}(\mathrm{pH} 7.5)$ for $3 \mathrm{~min}$ at $95^{\circ} \mathrm{C}$ and slowly cooled to $23^{\circ} \mathrm{C}$ in $15 \mathrm{~min}$. Selfcleavage reactions in the presence or the absence of inhibitory compounds were triggered by adding $\mathrm{MgCl}_{2}$ to $1 \mathrm{mM}$ or $10 \mathrm{mM}$ at $23^{\circ} \mathrm{C}$. The reaction was stopped by adding 5 volumes of stop solution ( $8 \mathrm{M}$ urea, $50 \%$ formamide, $40 \mathrm{mM}$ EDTA), and the cleaved fragments were separated by a $10 \%$ denaturing polyacrylamide gel. Cleavage percentage was then quantified by PhosphorImager. We did not detect any cleavage products in transcription reactions when tubercidin triphosphate was used to replace ATP.

\section{ACKNOWLEDGMENTS}

We thank Jack Szostak for helpful discussions and for reviewing the manuscript, Steve Gullans for providing the FDA2000 library, and Yi Tang for assistance with bioluminescence imaging. This work was supported by grants from AMGEN and L'Association Francaise contre les Myopathies (AFM) to R.C.M. and R24 CA92782 to R.W. R.C.M. is an AMGEN consultant and equity holder. B.R.S. was supported in part by a Career Award at the Scientific Interface and by the National Cancer Institute (1R01CA097061).

Received November 21, 2005; accepted January 13, 2006.

\section{REFERENCES}

Acs, G., Reich, E., and Mori, M. 1964. Biological and biochemical properties of the analogue antibiotic tubercidin. Proc. Natl. Acad. Sci. 52: 493-501.

Aszalos, A., Lemanski, P., Robison, R., Davis, S., and Berk, B. 1966. Identification of antibiotic 1037 as toyocamycin. J. Antibiot. (Tokyo) 19: 285.

De la Pena, M., Gago, S., and Flores, R. 2003. Peripheral regions of natural hammerhead ribozymes greatly increase their self-cleavage activity. EMBO J. 22: 5561-5570.

el Kouni, M.H. 1991. Efficacy of combination therapy with tubercidin and nitrobenzylthioinosine $5^{\prime}$-monophosphate against chronic and advanced stages of schistosomiasis. Biochem. Pharmacol. 41: 815-820.

el Kouni, M.H. and Cha, S. 1987. Metabolism of adenosine analogues by Schistosoma mansoni and the effect of nucleoside transport inhibitors. Biochem. Pharmacol. 36: 1099-1106. 
Ferbeyre, G., Smith, J.M., and Cedergren, R. 1998. Schistosome satellite DNA encodes active hammerhead ribozymes. Mol. Cell. Biol. 18: 3880-3888.

Fire, A., Xu, S., Montgomery, M.K., Kostas, S.A., Driver, S.E., and Mello, C.C. 1998. Potent and specific genetic interference by double-stranded RNA in Caenorhabditis elegans. Nature 391: 806-811.

$\mathrm{Fu}$, D.J. and McLaughlin, L.W. 1992. Importance of specific adenosine N7-nitrogens for efficient cleavage by a hammerhead ribozyme. A model for magnesium binding. Biochemistry 31: 10941-10949.

Glazer, R.I. and Lloyd, L.S. 1982. Association of cell lethality with incorporation of 5-fluorouracil and 5-fluorouridine into nuclear RNA in human colon carcinoma cells in culture. Mol. Pharmacol. 21: $468-473$.

Gossen, M. and Bujard, H. 1992. Tight control of gene expression in mammalian cells by tetracycline-responsive promoters. Proc. Natl. Acad. Sci. 89: 5547-5551.

- 2002. Studying gene function in eukaryotes by conditional gene inactivation. Annu. Rev. Genet. 36: 153-173.

Hammann, C., Norman, D.G., and Lilley, D.M. 2001. Dissection of the ion-induced folding of the hammerhead ribozyme using $19 \mathrm{~F}$ NMR. Proc. Natl. Acad. Sci. 98: 5503-5508.

Hermann, T. and Westhof, E. 1998. Aminoglycoside binding to the hammerhead ribozyme: A general model for the interaction of cationic antibiotics with RNA. J. Mol. Biol. 276: 903-912.

Hertel, K.J., Pardi, A., Uhlenbeck, O.C., Koizumi, M., Ohtsuka, E., Uesugi, S., Cedergren, R., Eckstein, F., Gerlach, W.L., Hodgson, R., et al. 1992. Numbering system for the hammerhead. Nucleic Acids Res. 20: 3252.

Jaffe, J.J., Meymarian, E., and Doremus, H.M. 1971. Antischistosomal action of tubercidin administered after absorption into red cells. Nature 230: 408-409.

Jenne, A., Hartig, J.S., Piganeau, N., Tauer, A., Samarsky, D.A., Green, M.R., Davies, J., and Famulok, M. 2001. Rapid identification and characterization of hammerhead-ribozyme inhibitors using fluorescence-based technology. Nat. Biotechnol. 19: $56-61$.

Kelley, B.P., Lunn, M.R., Root, D.E., Flaherty, S.P., Martino, A.M., and Stockwell, B.R. 2004. A flexible data analysis tool for chemical genetic screens. Chem. Biol. 11: 1495-1503.

Khvorova, A., Lescoute, A., Westhof, E., and Jayasena, S.D. 2003. Sequence elements outside the hammerhead ribozyme catalytic core enable intracellular activity. Nat. Struct. Biol. 10: 708712.

Longley, D.B., Harkin, D.P., and Johnston, P.G. 2003. 5-Fluorouracil: Mechanisms of action and clinical strategies. Nat. Rev. Cancer 3: 330-338.

Lunn, M.R., Root, D.E., Martino, A.M., Flaherty, S.P., Kelley, B.P., Coovert, D.D., Burghes, A.H., Man, N.T., Morris, G.E., Zhou, J., et al. 2004. Indoprofen upregulates the survival motor neuron protein through a cyclooxygenase-independent mechanism. Chem. Biol. 11: 1489-1493.

McManus, M.T. and Sharp, P.A. 2002. Gene silencing in mammals by small interfering RNAs. Nat. Rev. Genet. 3: 737-747.

Murray, J.B. and Arnold, J.R. 1996. Antibiotic interactions with the hammerhead ribozyme: Tetracyclines as a new class of hammerhead inhibitor. Biochem. J. 317: 855-860.

Penedo, J.C., Wilson, T.J., Jayasena, S.D., Khvorova, A., and Lilley, D.M. 2004. Folding of the natural hammerhead ribozyme is enhanced by interaction of auxiliary elements. RNA 10: $880-888$.

Pley, H.W., Flaherty, K.M., and McKay, D.B. 1994. Three-dimensional structure of a hammerhead ribozyme. Nature 372: 68-74.

Rivera, V.M., Clackson, T., Natesan, S., Pollock, R., Amara, J.F., Keenan, T., Magari, S.R., Phillips, T., Courage, N.L., Cerasoli Jr., F., et al. 1996. A humanized system for pharmacologic control of gene expression. Nat. Med. 2: 1028-1032.

Rogers, J.T., Randall, J.D., Eder, P.S., Huang, X., Bush, A.I., Tanzi, R.E., Venti, A., Payton, S.M., Giordano, T., Nagano, S., et al. 2002. Alzheimer's disease drug discovery targeted to the APP mRNA 5'untranslated region. J. Mol. Neurosci. 19: 77-82.

Root, D.E., Flaherty, S.P., Kelley, B.P., and Stockwell, B.R. 2003. Biological mechanism profiling using an annotated compound library. Chem. Biol. 10: 881-892.

Ross, A.G., Bartley, P.B., Sleigh, A.C., Olds, G.R., Li, Y., Williams, G.M., and McManus, D.P. 2002. Schistosomiasis. N. Engl. J. Med. 346: 1212-1220.

Scott, W.G., Finch, J.T., and Klug, A. 1995. The crystal structure of an all-RNA hammerhead ribozyme: A proposed mechanism for RNA catalytic cleavage. Cell 81: 991-1002.

Stage, T.K., Hertel, K.J., and Uhlenbeck, O.C. 1995. Inhibition of the hammerhead ribozyme by neomycin. RNA 1: 95-101.

Suhadolnik, R.J., Uematsu, T., and Uematsu, H. 1967. Toyocamycin: Phosphorylation and incorporation into RNA and DNA and the biochemical properties of the triphosphate. Biochim. Biophys. Acta 149: 41-49.

Suhr, S.T., Gil, E.B., Senut, M.C., and Gage, F.H. 1998. High level transactivation by a modified Bombyx ecdysone receptor in mammalian cells without exogenous retinoid X receptor. Proc. Natl. Acad. Sci. 95: 7999-8004.

Tor, Y., Hermann, T., and Westhof, E. 1998. Deciphering RNA recognition: Aminoglycoside binding to the hammerhead ribozyme. Chem. Biol. 5: R277-R283.

Wang, Y., O'Malley Jr., B.W., Tsai, S.Y., and O’Malley, B.W. 1994. A regulatory system for use in gene transfer. Proc. Natl. Acad. Sci. 91: 8180-8184.

Yen, L., Svendsen, J., Lee, J.S., Gray, J.T., Magnier, M., Baba, T., D'Amato, R.J., and Mulligan, R.C. 2004. Exogenous control of mammalian gene expression through modulation of RNA selfcleavage. Nature 431: 471-476. 

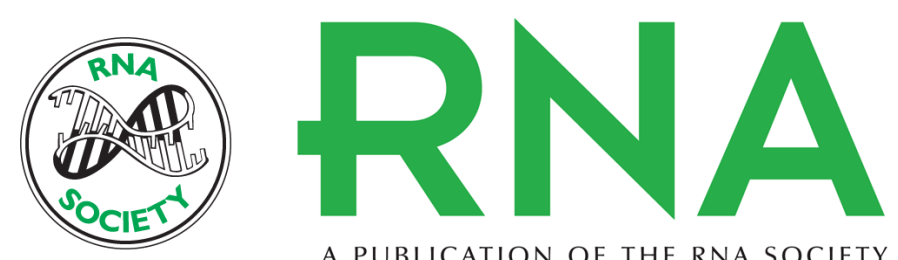

A PUBLICATION OF THE RNA SOCIETY

\section{Identification of inhibitors of ribozyme self-cleavage in mammalian cells via high-throughput screening of chemical libraries}

Laising Yen, Maxime Magnier, Ralph Weissleder, et al.

RNA 2006 12: 797-806

References This article cites 35 articles, 11 of which can be accessed free at:

http://rnajournal.cshlp.org/content/12/5/797.full.html\#ref-list-1

License

Email Alerting Receive free email alerts when new articles cite this article - sign up in the box at the Service top right corner of the article or click here. 